\title{
Proximal Focal Femoral Deficiency, Contralateral Hip Dysplasia in Association with Contralateral Ulnar Hypoplasia and Clefthand: A Case Report and Review of Literatures of PFFD and/or FFU
}

\author{
By \\ Ahmet KALAYCIOGLU, MD, PhD* and Osman AYNACI, MD** \\ * Department of Anatomy, Faculty of Medicine, Karadeniz Technical University, Trabzon, Turkey \\ ** Department of Orthophedics, Faculty of Medicine, Karadeniz Technical University, Trabzon, Turkey \\ - Received for Publication, February 4, 2000 -
}

\begin{abstract}
Key Words: Proximal focal femoral deficiency (PPFD), Congenital deficiency of femur (CDF), Femoral hypoplasia, Short femur, Femur-fibula-ulna complex (FFU), Abnormalities extremities

Summary: A 14 year old boy with proximal focal femoral deficiency (PFFD) on left side, contralateral hip dysplasia in association with ulnar hypoplasia and cleft hand was seen our clinic one year ago. From our research, despite it is atypical presentation, this case in a broad context conforms with the femoral-fibula-ulna complex (FFU). This boy was born to healthy and nonconsanguineous parents. Pregnancy and delivery were uncomplicated, and no history of prenatal teratogen exposure, any drug ingestion, infection, diabetes mellitus or other conditions that could be associated with malformation. The patient refused any sort of surgical treatment.
\end{abstract}

The term FFU complex has been proposed for cases in which the femur-fibula and/or ulna show defects, which tend to be associated ${ }^{36}$.

Some cases show changes typical of FFU complex only on the arms. The legs need not to be affected. Even cases in which only one arm or one leg is affected may still belong to the same category ${ }^{36}$.

Ulnar hypoplasia or agenesis is a much rarer disorder than its usual structural companion, longitudinal deficiency of the radius. Its even rarer when accompanied with contralateral proximal focal femoral deficiency, and to data very few documentations have been recorded of such cases ${ }^{36,46)}$.

The original description of the femur-fibula-ulna (FFU), complex a malformation syndrome that usually occurs sporadically, mentioned the absence of the proximal part of the femur, absence of the fibula and malformations of the ulnar side of the upper limb ${ }^{36}$. Later cases of bilateral femur and fibula defects with normal arms were included in the same category ${ }^{37)}$.

Congenital longitudinal deficiency of the ulna is one of the rarest of the upper limb anomalies, ranking second to absence of the humerus ${ }^{46)}$. Ac- cording to Tachdjian, Birch-Jensen reported, its incidence is 1 in 100.000 live births ${ }^{46}$. The anomaly was first described by Goller in 1693. The defect is referred to in the literature by various terms ${ }^{46}$.

Proximal focal femoral deficiency is a high incidence of associated anomalies; $69 \%$ in the series reported by Aitken and $65 \%$ of those reported by Koman. Congenital longitudinal deficiency of the fibula is the most common associated anomaly, its incidence reported as $50 \%$ by Nilsonne ${ }^{46)}$. Some investigators reported that longitudinal deficiency of the tibia associated with fibular dimelia was a very rare condition ${ }^{34.40)}$. Congenital longitudinal deficiency of the fibula is not an isolated malformation. The fibular deficiency ranges from partial absence to complete absence. The tibia is almost always shortened and, in complete aplasia of the fibula. The right side is more often involved than the left. Occasionally, involvement is bilateral. It is more prevalent in boys than in girls ${ }^{46}$.

In result; the types of malformations that occured was analysed in two groups;

1-Patients with one limb affected

2-Patients with more than one limb affected ${ }^{361}$. 


\section{Observation}

This 14 year old male patient was first seen at our clinic a year ago. On examination, he appeared alert with normal mental development and no history of visceral or internal organs abnormality. His right upper and left lower extremities were observed to be shorter than their contralateral sides, in addition to the absence of the ulnar ray in the right upper extremity.

Orthopedic examination of his thorax confirmed a normal appearance. His right upper extremity in contrast to the left was shorter, with contractual flexion at the elbow and the presence of only the first and second fingers. The left upper extremity was normal. The left thigh was foreshortened in a position of flexion, abduction and external rotation. Its distal segment appeared grossly abnormal, disproportionately shorter and thinner than that of the right leg. The right hip and thigh appeared normal with a minimal degree of bowing of the femur. The ankles of joints and feet of both extremities were normal (Fig. 1).

Roentgenograms revealed humeral radial synostosis of the right elbow joint, bowing of the radius and hypoplasia of the ulna in addition to absence of the right ulna ray of the carpals, metacarpals and phalanges was noted (Fig. 2). The right hip was shallow, but the left hip showed on Aitken Type C proximal focal femoral deficiency (PFFD). The right femur was intact and clearly longer than the left femoral segment with a coxa vera determity (Fig. 3). The tibia and fibulas of both lower extremities were normal.

The patient was born to healthy and nonconsanguineous parents. Pregnancy and delivery were uncomplicated, without contact with teratogenic agents. There was no history of congenital anomalies in the family. Results of chromosome analysis were normal.

\section{Results}

Despite the fact that the FFU complex had to include defects of the femur when first proposed, fibula and/or ulna, today it has a broad context of a defect of the ulna or fibula alone. In addition, it demonstrates a prominantly sporadic occurrence without a genetic factor tends to be more asymmetric, and has a preponderancy in the male population. Our patient has many respects which fulfills criteria of this complex. According to Anderson et al. and Kostuik, Syme amputation is not always effective. The major indication was leg length discrepancy, due to either paraxial fibula hemimelia or

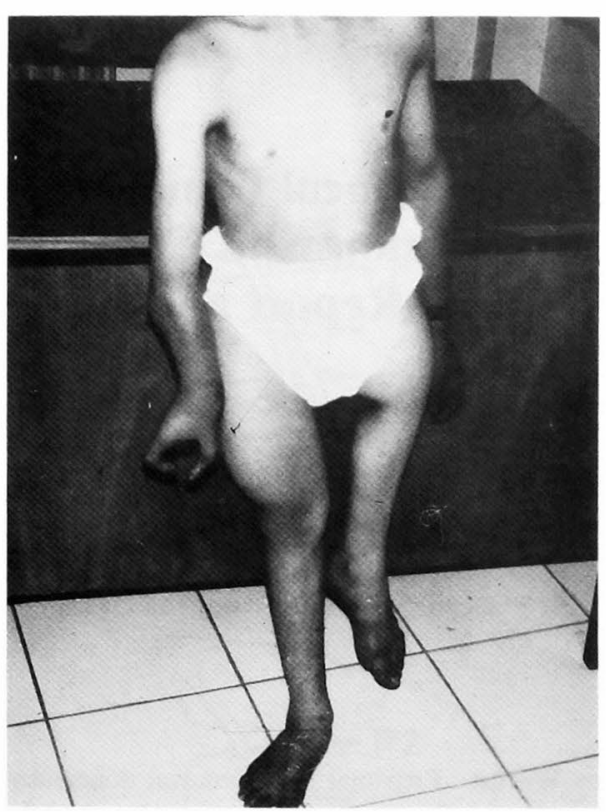

Fig. 1. Photograph of children.

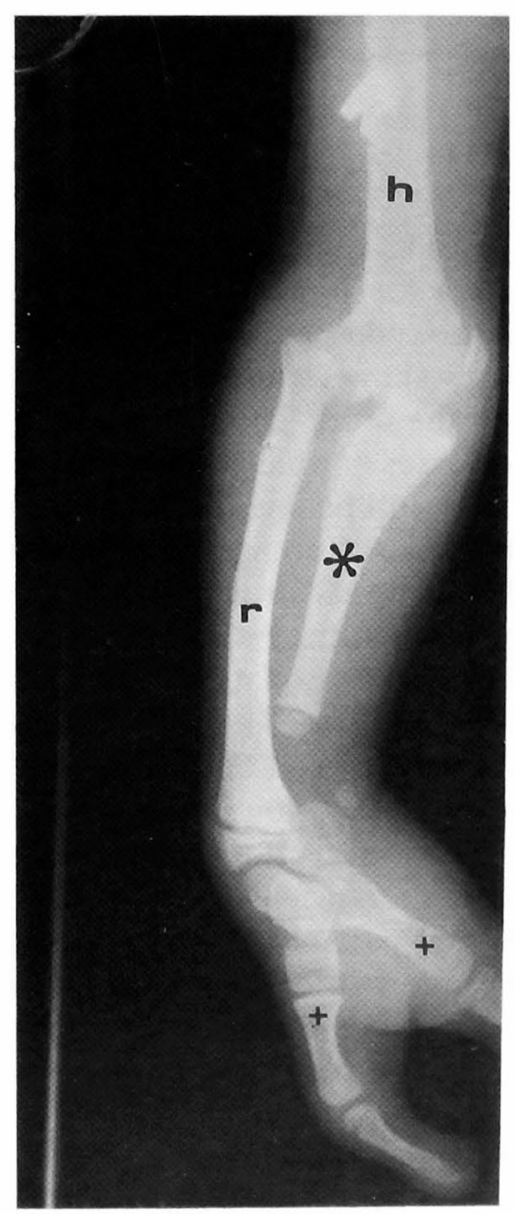

Fig. 2. Abnormality of right upper limb (ulna). h: humerus, r: radius, *: hypoplasia of ulna, + : phalanges of cleft hand. 


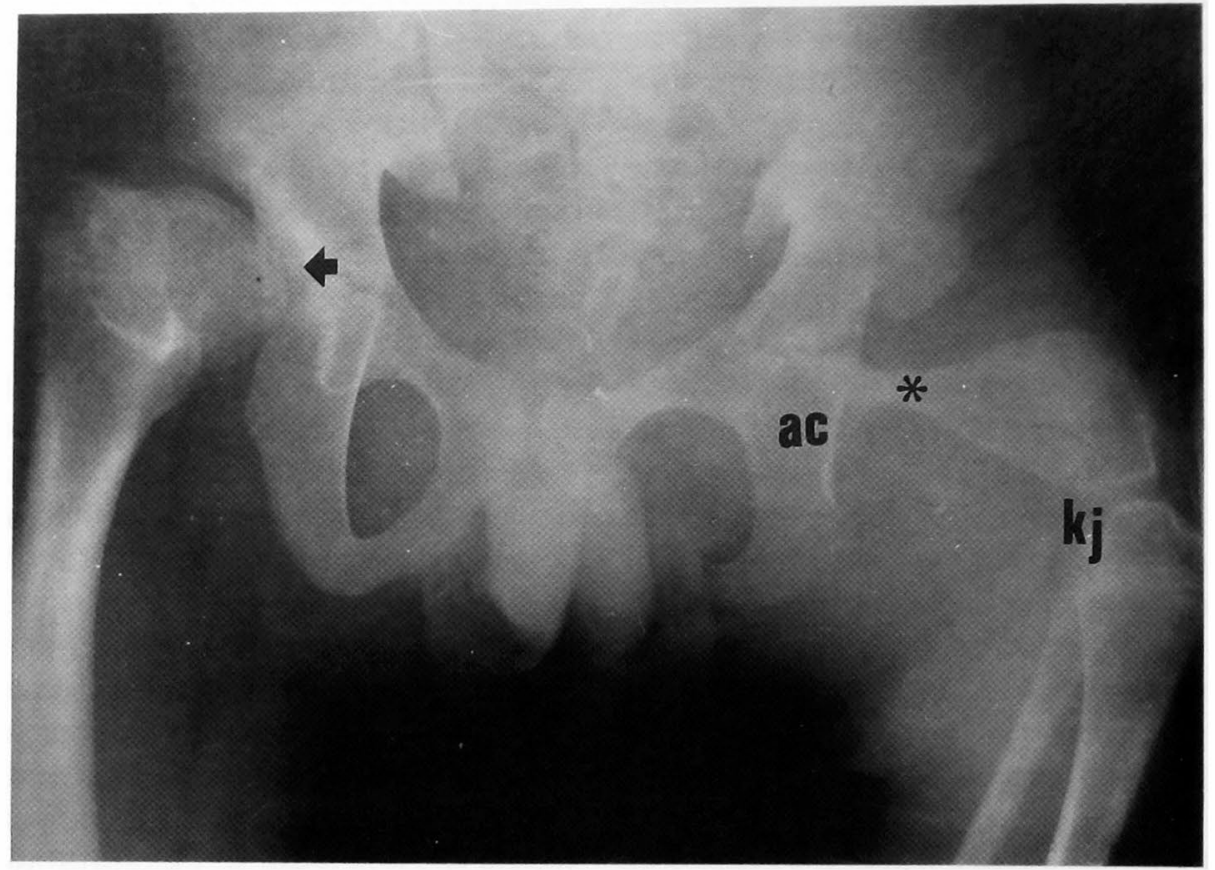

Fig. 3. Abnormal head and shaft of left femur and right displastic hip joint. ac: acetabulum, *: proximal focal deficiency of femur, kj: knee joint, $\rightarrow$ : displastic hip joint on right.

proximal focal femoral deficiency. Prosthetic knees were often too low, because of failure to limit the length of the stump appropriately ${ }^{4,33)}$. Congenital synostosis of elbow is very rare abnormality and it may occur either as an isolated deformity or with other anomalies such as absence of the ulna, or fusion/absence of the carpals, metacarpales or phalanges. In the isolated form, involement is usually bilateral, whereas in the associated form, it may be unilateral or bilateral. There is no sex predilection. The ankylosis may occur at the humeroradial, humeroradioulnar, or humeroulnar joints. In humeroulnar or humeroradial synostosis, the radius or ulna is usually absent. Functional disability depends on the position in which the elbow is fixed. In unilateral ankylosis, it is best to perform an osteotomy to achieve a functional position. Surgical correction in late childhood can prevent disuese atrophy of the musculature ${ }^{46)}$. The benefits of deformity of $\mathrm{lcg}$ lengthening in younger children include early correction of deformity and a technically easier procedure $^{241}$. In diagnostic use of CT scans and MRI have proved to have great diagnostic and prognostic values.

\section{Discussion}

The precise diagnosis of FFU complex is particularly difficult because cases which belong to this complex can differ widely from one to another. The term FFU complex was originally proposed to be the cases with femur, fibula and/or ulna show defects, which tend to be associated and usually sporadic $^{36}$. Lenz and Feldman reported that FFU in its broad context includes defects of fibula or ulna alone. There is striking asymmetry in presence and in degree. All malformations are more often unilateral than bilateral. Upper limbs are affected more often than lower limbs, with the right side and male are preferencially affected ${ }^{37)}$. As the limb malformations present in the FFU complex are different from those seen in other type of limb defects, there is virtually no overlap between FFU complex and other limb malformations. Such anomalies include, amelia, peromelia of humerus, humeroradial synostosis and defects of the ulna or ulna rays. FFU syndrome is not associated with internal malformations and familial recurrency is quite unusual, when compared to extremity to malformations in genetic syndromes ${ }^{36)}$. One investigation reported 114 cases with isolated ulnar-fibular deficiency in Hungary, 1975-1984. The investigators said that ulnar-fibular type had the third most common birth prevalance among isolated congenital limb deficiency types and they were correlated with lower birth weight, higher birth order, lower socioeconomic status of parents, and a more frequent reported subfertility $^{12)}$.

In some familial cases, the brain or the face is 
malformed; therefore, FFU complex was excluded in some cases ${ }^{36}$. Familial cases have been described by some investigators ${ }^{6,11,32)}$. A clinical syndrome including femoral hypoplasia and unusual facial appearance was reported ${ }^{6,29)}$. For example, Hersh et al., reported nasal abnormalities with dimelia of ulna and fibula, tibial hypoplasia and mirror image duplication of hands and feet ${ }^{21)}$. A case was reported with bilateral femoral hypoplasia associated with rokitansky sequence ${ }^{6)}$. Some authors described congenital round talus correlation with FFU-syndrome ${ }^{8)}$ and congenital ball-and-socket anomaly of the ankle correlation with FFUsyndrome ${ }^{\text {9). }}$.

Gonzales et al. reported a cases two families with an autosomal dominant syndrome of limb and hematologic abnormalities ${ }^{17)}$. Lipson described a male infant with symmetrical tetramelic limb deficiency consisting of bilateral upper limb amelia with severe symmetrical proximal focal femoral deficiency and fibula deficiency associated with left splenogonal fusion of the discontinuous type, micrognathia and a prominent capillary hemangioma of the face ${ }^{38)}$, and Kardon et al. reported, the amelia/phocomelia with facial hemangiomas and micrognathia ${ }^{31)}$. Robert et al. reported that in proximal femoral focal deficiency (PFFD) or FFU complex an hereditary factor does not play a role. Neither iatrogenic agent, radiation exposure, nor infectious diseases can incriminated by a study in the Rhono-Alpesregion, in France $1979^{43}$ ). Infants born to diabetic mothers have a higher frequency of skeletal malformations. They are prone to have delayed ossification ${ }^{23)}$. Among the fetal anomalies related to maternal diabetes, the caudal regression syndrome, defined as agenesis or hypoplasia of the femur with or without the agenesis of the lower vertebrae, is the best known for its high relative risk ratio of over $200^{23}$. In addition to skeletal dispylasias, a short femur and humerus have been associated with chromosomal anomalies such as Down syndrome ${ }^{7)}$.

Congenital anatomic abnormalities of the femur were reported and reviewed by several investigators. Those ranged from the femur approximately $60 \%$ of the lenght of the normal leg to the subtotal absence of the femur in which only the femoral condyles remained ${ }^{15)}$.

The first description was by Otto in 1841 , more than fifty years ago before the discovery of roentgenograms. Associated anomalies are common and have included ipsilateral bifurcation of femur ${ }^{15}$. The proximal focal femoral deficiency (PFFD) may be unilateral or bilateral and is often associated with other congenital anomalies ${ }^{18,35)}$. The incidence of this complex defect has been reported by Rogala et al. (1974) from Edinburg Register of the Newborn to be about 1 in 50.000 of population ( $0,2 \mathrm{Per}$ $10.000)^{18)}$. In modern orthopedic literature, incomplete duplication of both femur and double acetabulum was reported in 1931 by Nitche ${ }^{25)}$. Fibular hemimelia has been reported in association with total duplication of the femur, but never with bifurcation of the femur ${ }^{18)}$. Ostrum et al. reported Burkus and Ogden's study: They had dissected 103 prenatal femurs and found one femur from a fetus of 7 weeks of gestation with two centers of primary ossification $^{41)}$. Bilateral absence of the radius and tibia with duplication of the ulna and fibula, have been reported by both Laurin $e t$ al. and Sandrow et al. who described the condition in a father and daughter ${ }^{25)}$.

Numerous extrinsic factors such as trauma, drugs, viral infection, irradiation, focal ischemia, a change of hormonal conditions and chemical toxicity have been considered as a cause, acting from the fourth to the eighth week of gestation, but none of them except the antidepressant drug Thalidomide has been confirmed as a definite aetiological factor $^{20)}$.

Johansson and Aparisi reported six cases of congenital absence of one or both cruciate ligaments of the knee in patients with mild proximal femoral focal deficiency. Radiographic examination showed changes in the tibial spine in all affected knees ${ }^{28)}$. Some investigators reported knee disarticulation or subluxation incidence increase, if it accompanied with congenital short femur $(\mathrm{CSF})^{40,44)}$.

The ulnar-mammary-syndrome (UMS) was described by Bamshad et al. The UMS is an autosomal dominant disorder characterised by posterior limb deficiencies or duplications, apocrine/mammary gland hypoplasia and/or disfunction, abnormal dentition, delayed puberty in males and genital anomalies. While most patients have limb deficiencies, the range of abnormalities extends from hypoplasia of the terminal phalanx of the digits to complete absence of the ulna. Moreover, affected individuals may have posterior digital duplications with or without contralateral limb deficiencies ${ }^{5)}$. The limbs show a great variety of absence malformations which are strikingly asymmetric in comparison with other genetic absence malformations. The limb defects are highly variable even within the same family. The pattern of the skin lesions corresponds to the Blaschko lines and may be explained by the Lyon effect due to random inactivation of one of two female $\mathrm{X}$ chromosomes. The variable bone defects may be attributed to the same mechanism, which produces a mosaic of normal and abnormal cells $^{36)}$. Many classification systems have been developed over the years. For PFFD (Proximal Focal 
Femoral Deficiency), the best known classifications are those of Ring $1959^{18)}$, Aitken $1969^{1)}$, Amstutz $1969^{3)}$, Fixen-Lloyd 1974 ${ }^{13)}$, Kostuik 1975 ${ }^{33)}$, Hamanishi 198020) (figüre 4), Pappas 198342), Gillespie-Torode 1983'5), Kalamchi $1985^{30}$, and Grissom-Harcke $1994^{19}$ ). Similar cases with our's were reported by Aitken in his Type $C$, Hamashi in his Type IV-h, by Pappas in his Class II.

According to Goldman et al., the term proximal focal femoral deficiency (PFFD) is applied to a spectrum of conditions characterised by partial absence and shortening of the proximal femora and thought to result from an early disturbance of growing mesenchyme. The mildest cases, Classes A and $B$, exhibit relatively normal acetabulum and capital femoral epiphysis, with the dysplastic shaft. There may be a subtrochanteric varus deformity or a pseudo arthrosis accompanying the shortening of the femoral shaft. At the opposite end of spectrum, the most severely deformed cases, Classes C and D, have involvement of the acetabulum and the entire proximal femur ${ }^{16)}$. Wolfgang reported from Salzer's review; 181 cases of tibial hemimelia from the world literature and he was reported $3.3 \%$ of congenital dislocation of the hip and $16 \%$ of proximal femoral focal defect, $7.2 \%$ of cleft hand, and $3.8 \%$ of radial hemimelia ${ }^{47)}$.

Ulnar ray deficiency were reviewed by Swanson et al. Based with the findings that a subclassification into 4 types were established; type I; hypoplasia or partial defect of the ulna, type II: total defect of the ulna, type III; total or partial defect of the ulna with humeroradial synostosis, and type IV; ulnar defect with congenital amputation at the wrist. Contral digits and carpal bones were also influenced by ulnar ray deficiency, presenting carpal bone fusion, syndactyly, and delta phalan $x^{45}$.

There is no evidence for monogenetic inheritance in regar to the aetyology of the FFU complex. The following arguments virtually exclude any simple genetic explanation.

1) The FFU complex shows a degree of asymmetry not usually found in genetic conditions.

2) FFD is typically sporadic and familial recurrence is exceptional and may be due to a chance.

3) No transmission from a parent to a child has been observed.

4) The rate of consanguinity is not increased among the parents ${ }^{36}$.

Chromosomal studies have failed to show any abnormalities and the complex has not been described as a part of any of the known deletion or duplication syndromes ${ }^{39)}$.

In previous studies on the FFU complex the most frequent combinations are as follows:

1. Femur-fibula, 2. Femur-fibula-ulna, 3. Fibulaulna, 4. Femur-ulna, 5. Fibula-toes ${ }^{36)}$.

Jago et al. reported that femur length measured
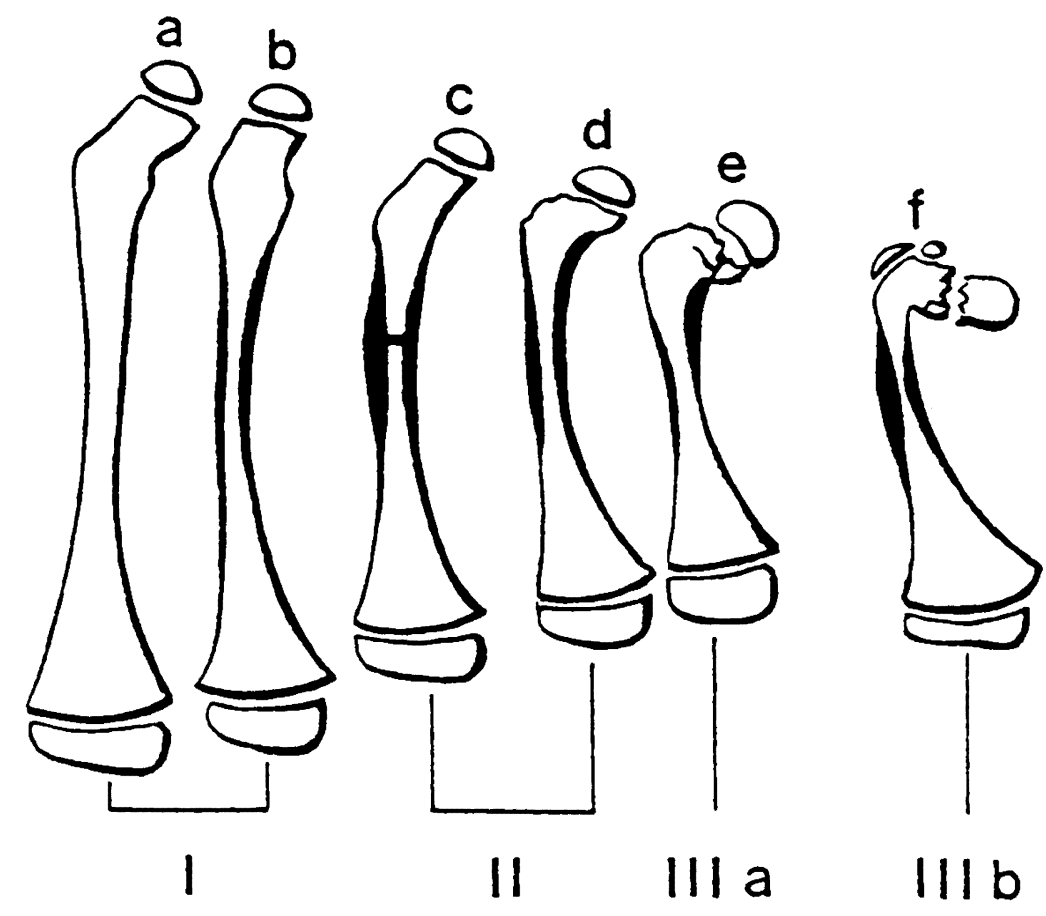

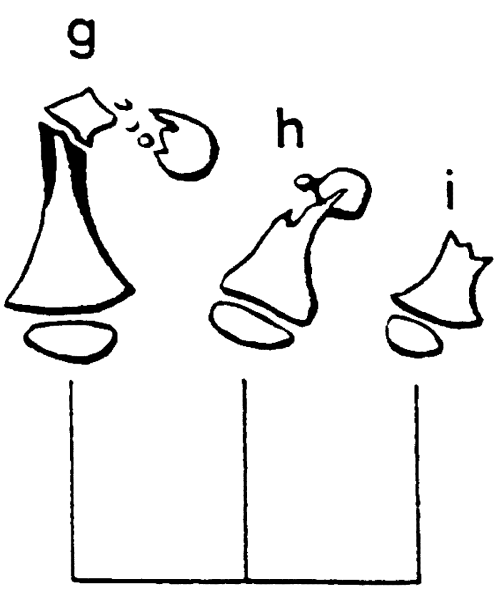

IV

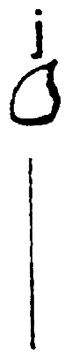

V

Fig. 4. Classification of congenital short femur from Hamanishi (Hamanishi C. Congenital short femur. The Journal of Bone and Joint Surgery 1980 Aug; Vol: 62-B, No: 3: 302-320). Reproduced with author's permission. 
by a typical modern machine was likely to appear to be about $1 \mathrm{~mm}$ shorter than the same femur measured bt an earlier generation machine ${ }^{26)}$. According to Hope et al., the benefits of lengthening leg with deformity in younger children include early determity and a technically easier procedure ${ }^{24)}$.

A case of ultrasonographic prenatal diagnosis at 24 weeks of femur-fibula-ulna (FFU) complex was presented ${ }^{10)}$. According to Hilmann et al., proximal focal femoral deficiency, uncommon congenital anomaly, needs early radiologic classification for surgical planning and treatment. Objective radiographic criteria should include femoral length index, acetabular depth index and acetabular angle inde $\mathrm{x}^{22)}$. Florio et al. said that the importance of an early diagnosis of FFU complex is emphasized with respect to parental counselling for prognosis and further prenatal management ${ }^{14)}$.

Recently the use of CT scans and MRI have proved to be of great diagnostic and prognastic value.

In treatment, different techniques were used for PFFD: Van Nes Rotation-plasty, rotation-plasty of the lower limb through the knee with simultaneous knee fusion technique of Torode-Gillespie, knee fusion for prosthetic conversion in proximal femoral focal deficiency technique of King, and Syme's amputation technique ${ }^{46)}$. According to Kostuik, Van Nes rotational osteotomy was not performed in patients with bilateral proximal femoral focal deficiency. Van Nes procedure should provide what a child with a Syme's amputation and a congenital short femur or proximal femoral focal deficiency does not have a foot upon which he can walk without a prosthesis if necessary, as well as active knee joint motion which allows participation is sports and makes climbings stairs in a normal fashion possible ${ }^{33)}$. Kostuik et al. believe that it is a mistake to do the Van Nes procedure before the patient is twelve years old. When it is done earlier, rerotation of the foot during the rapid growth period will be inevitable. A failed van Nes rotational osteotomy is not a procedure from which there is no recourse. A Syme's amputation can still be performed to salvage the situtation ${ }^{33)}$. Jayakumar and Eilert reported in patients with congenital abcence of the tibia, construction of a knee joint by transferring the fibula under femoral condyle gives satisfactory results. If a strong quadriceps was present, the procedure could be performed as early as the first year of life ${ }^{27)}$. According to Alman et al., the treatment of isolated unilateral proximal femoral focal deficiency (PFFD) with rotationplasty resulted in a more energy efficient gait than the treatment with Syme amputation, with no difference in percived physical appearence or gross function ${ }^{2}$.

\section{References}

1) Aitken GT. Proximal femoral focal deficiency-definition classification, and management. In: Aitken GT. ed. Proximal femoral focal deficiency: A congenital anomaly. Washington D.C., National Academy of Sciences 1969; 1-22.

2) Alman BA, Krajbich JI and Hubbard S. Proximal femoral focal deficiency. Result of Rotationplasty and Syme Amputation. J Bone Joint Surg 1995 Dec; Vol: 77A, No: 12:1876-1882.

3) Amstutz HC. The morphology, natural history and treatment of proximal femoral focal deficiency, In: Aitken GT. ed. Proximal femoral focal deficiency. A congenital anomaly. Washington D.C.; National Academy of Science 1969; 50-76.

4) Anderson L, Westin GW and Oppenheim WL. Syme amputation in children, indications, results and long term follow up. J Pediatr Orthop 1984 September; 4(5):550-4.

5) Bamshad M, Root S and Corey JS. Clinical analysis of a large kindred with the Pallister ulnar-mammary syndrome. Am J Med Genet. 1996 Nov; 11, 65-4:325-31.

6) Bau CHD, Riberio CA, Riberio SA and Flores RZ. Bilateral Femoral Hypoplasia Associated With Rokitansky Sequance: Another example of a mesodermal spectrum. Am J Med Genet 1994; 49:205-206.

7) Benacerraf BR, Neuberg D and Frigoletto FD (Jr). Humoral shorthening in second trimester fetuses with Down Syndrome. Obstet Gynecol 1991 Feb; 77(2):223-227.

8) Bettin D and Karbowski A. Congenital round talus. $Z$ Orthop Ihre Grenzgeb 1995 Jan-Feb; 133(1):50-54.

9) Bettin D, Karbowski A and Schwering L. Congenital ball and socket anomaly of the ankle. J Pediatr Orthop 1996 Jul-Aug; 16(4):492-496.

10) Capece G, Fasolino A, Della Monica M, Lonardo F, Scarano $G$ and Neri G. Prenatal diagnosis of femur-fibulaulna complex by ultrasonography in a male fetus at 24 weeks of gestation. Prenat Diagn 1994 Jun; 14(6):502-505.

11) Connor JM, Rae PS and Connor RA. Monozygotic twins concordant for congenital short femur. J Med Genet 1986 Aug; 23(4):363-364.

12) Czeizel AE, Vitez M, Kodaj I and Lenz W. Causal study of isolated ulnar-fibular deficiency in Hungary, 1975-1984. Am J Med Genet 1993 Jun; 1; 46(4):427-433.

13) Fixen JA and Lloyd-Roberts GC. The natural history and early treatment of proximal femoral dysplasia. J Bone Joint Surg (Br) $1974 \mathrm{Feb} ;$ 56:B, No: 1:86-95.

14) Florio I, Wisser $J$ and Huch A. Prenatal Ultrasound Diagnosis of a Femur-Fibula-Ulna Complex during the First Half of Pregnancy. Fetal Diagn Ther 1999 Oct; 14(5):310 312.

15) Gillespie R and Torode IP. Classification and management of Congenital Anomalies of the Femur. J Bone Joint Surg 1983 November; Vol: 65-B, No: 5:557-568.

16) Goldman AB, Schneider R and Wilson PD (Jr). Proximal focal femoral deficiency. J Can Assoc Radiol 1978 June; 29(2):101-7.

17) Gonzales $\mathrm{CH}$, Durkin-Stamn MV, Geimer NF, et al. The WT syndrome a-"new" autosomal dominant pleitropic trait of radial/ulnar hypoplasia with high risk of bone marrow failure and/or leukemia. Birth Defects 1977; 13(3B):31-38.

18) Grill $F$ and Dungl $P$. Lengthening for congenital short femur. Result of Different Methods. J Bone Joint Surg (Br) 1991 May; 73(3):439-447.

19) Grissom LE and Harcke HT. Sonography in Congenital Deficiency of the Femur. J Pediatr Orthop 1994; 14:29-33.

20) Hamanishi C. Congenital short femur: clinical, genetic and epidemiological comparison of the naturally occuring con- 
dition with that caused by Thalidomide. J Bone Joint Surg (Br) 1980 August; 62-B, No: 3:307-320.

21) Hersh JH, Dela Cruz TV, Pietrantoni M. et al. Mirror image duplication of the hands and feet: report of a sporadic case with multiple congenital anomalies. Am J Med Genet 1995 Nov 20; 59(3):341-345.

22) Hilman JS, Mesgarzadeh M, Revesz G, Bonakdarpour A, Clancy $M$ and Betz RR. Proximal femoral focal deficiency; radiologic analysis of 49 cases. Radiology 1987 Dec; 165(3):769-773.

23) Hitti IF, Glasberg SS, Jones DH and Sabet R. Bilateral femoral hypoplasia and maternal diabetes mellitus. Pediatr Pathol 1994; 14:567-574.

24) Hope PG, Crawfurd EJP, Catteral A and Chir M. Bone Graft Following Lenthening for Congenital Shorthening of the Lower Limb. J Pediatr Orthop 1994; 14:339-342.

25) Igous RA (Jr) and Kruger LM. Fibula Dimelia in Association With Ipsilateral Proximal Focal Femoral Deficiency and Polydactyly, A case report. Clin Orthop 1990 Sep; No: 258:237-241.

26) Jago JR, Whittingam TA and Heslop R. The influence of ultrasound scanner beam with on femur length measurements. Ultrasound in Med and Biol 1994; Vol: 20, No: 8:669-703.

27) Jayakumar SS and Eilert RE. Fibular transfer for congenital absence of the tibia Clin Ortop 1979 Mar-Apr; 139:97101.

28) Johanson E and Aparisi T. Missing cruciate ligament in congenital short femur. J Bone Joint Surg Am 1983 Oct; Volume 65(8):1109-15.

29) Johnson JP, Carey JS, Gooch 3WM 3d, Petersen J and Beattie JF. Femoral hypoplasia unusual faces syndrome in infants of diabetic mother. J Pediatr 1983; 102(6):866-872.

30) Kalamchi A, Cowell HR and Kim KI. Congenital deficiency of the femur. J Pediatr Orthop 1985 Mar-Apr; 5(2):12934.

31) Kardon NB, Dana LP, Fitz Gerald JM and Opitz JM. Two sporadic cases of amelia/phocomeelia with similar phenotype: rare and unusually symmetrical form of FFU dysostosis or separate entity. Am J Genet Suppl 1986; 2:239-245.

32) Keret $D$ and Timor IE. Pediatric Update; Familial congenital short femur, intrauterine detection and follow up by ultrasound, a case report. Orthop 1993 Mar-Apr; 13(2):203-209.

33) Kostuik JP, Gillespie R, Hall JE and Hubbard S. Van Nes Rotational Osteotomy for Treatment of Proximal Femoral
Focal Deficiency and Congenital Short Femur. J Bone Joint Surg 1975 December; Vol: 57-A, No: 8:1039-1046.

34) Kumar A and Kruger LM. Fibular dimelia with deficiency of the tibia. J Pedatr Orthop 1993 Mar-Apr; 13(2):203209.

35) Kurtz AB, Nedleman L, Wegner RJ, et al. Usefulness of a short femur in the in utero detection of skeletal dysplasias. Radiology 1990; 177:197-200.

36) Lenz W, Zygulska $M$ and Herst J. FFU complex; an analysis of 491 cases. Hum. Genet. 1993; 91:347-356.

37) Lenz W and Feldman U. Unilateral and asymetric limb defects in man. Delineation of the femur fibula ulna complex. Birth Defects 1997; 13:269-285.

38) Lipson AH. Amelia of the arms and femur/fibula deficiency with splenogenodal fusion in a child born to a consanguineous couple. Am J Med Genet 1995 Jan 30; 55(3): 265-8.

39) Mc Kusick VA, Francomano CA, Antonarakis SE and Pearson PL. Mendelian Inhetitance In Man, A Catalog Of Human Genes And Genetic Disorders, Elevent Edition, Volüme 2, The Jons Hopkins University Press, 1994: 1812.

40) Mehrotra G, Bhargava SK, Shiv VK and Taneja K. Proximal focal femoral deficiency. Indian Pediatr 1993 Feb; 30(2):241-245.

41) Ostrum RF, Betz RR, Clancy $M$ and Stell HH. Bifurcated Femur With a Normal Tibia and Fibula. J Pediatr Orthop 1987; 7:224-226.

42) Pappas AM. Congenital abnormalities of femur and related lower extremity malformations. J. Pediatr Orthop 1983 Feb; 3(1):45-60.

43) Robert JM, Guibaud P and Robert E. A local outbreak of femoral hypoplasia or aplasia and femoral fibula-ulnarcomplex. J Genet Hum 1981 Dec; 29(4):379-394.

44) Sanpera I (Jr), Fixen JA, Sparks LT and Hill RA. Kneee in congenital short femur. J. Pediatr Orthop B 1995; 4(2):159 163.

45) Swanson AB, Tada $\mathrm{K}$ and Yanenobu K. Ulnar ray deficiency: its various manifestations; J Hand Surg (Am) 1984. Sep; 93:658-64.

46) Tachdjian MO. Pediatric orthopedics. Volume 1, Secon Edition, Philadelphia: W.B. Saunders Company 1990; 206 209, 561-564, 620-621.

47) Wolfgang GL. Complex Congenital Anomalies of the Lower Extremities; Femoral Bifurcation, Tibial Hemimelia and Diastasis of the Ankle. J Bone Joint Surg 1984 March: Vol: 66-A, No: 3:453-458. 